\title{
CHRONOLOGY OF NEPHROGENIC EVENTS IN STAGED ABORTED HUMAN EMBRYOS AND FETUSES
}

\section{Subhadra Devi Velichety ${ }^{1}$, K. Thyagaraju ${ }^{* 2}$, Siva Kumar Vishnubhotla ${ }^{3}$, B. Ravindra} Kumar ${ }^{4}$.

${ }^{1}$ Professor and Head, Department of Anatomy, Sri Padmavathi Medical College for Women, SVIMS University, Tirupati, Andhra Pradesh, India.

*2 Assistant Professor, Department of Anatomy, Sri Padmavathi Medical College for Women, SVIMS University, Tirupati, Andhra Pradesh, India.

${ }^{3}$ Senior Professor, Department of Nephrology, Sri Venkateswara Institute of Medical Sciences, Tirupati, Andhra Pradesh, India.

${ }^{4}$ Lecturer, Department of Anatomy, Sri Padmavathi Medical College for Women, SVIMS University, Tirupati, Andhra Pradesh, India.

\section{ABSTRACT}

Introduction: The description given in various textbooks and literature on development of nephrons in human kidney doesn't include details of chronology of nephrogenic events at various fetal ages. Though several studies were reported the knowledge on development of kidney especially on the nephrogenesis are limited. The studies emphasize the relationship between prenatal development of kidney and adult onset of renal diseases. Hence, an attempt was made in this study to obtain information by observing the serial sections of kidney of embryos and foetuses of different gestational ages for better understanding of nephrogenic events.

Material and methods: Thirty-five aborted embryos and dead fetuses of 5 weeks gestational age to full term were utilized for this study. The specimens were subjected to routine tissue processing and haematoxylin and eosin (H\&E) staining. 5 embryos of less than 8 weeks gestational age were processed as a whole and were serially sectioned. The histological sections of 5 microns thickness were observed for the time of appearance of various nephrogenic components and photographed.

Results: Differentiating pronephric, mesonephric and metanephric components in different weeks i.e. $05-12,13$ - 24, 25-36 were studied. In $06-12$ weeks group a delay in the appearance of Pro and mesonephric, Meso and metanephric ducts were observed that appeared during the 6 th week. Differentiation of other components have not completed by 6th week when compared with literature. In $13-24$ weeks also there is delay in corticomedullary differentiation that was observed at $16 \mathrm{wks}$. at which time the morphologically recognizable Nephrons were also observed. Major part of development occurred between 16-28 weeks instead of 16-24weeks as stated in the literature. Ampulla division continued beyond 24 weeks. Increased number of mature nephrons were observed between 24-28 weeks instead of 16-20 wks., nephron arcades were observed during 24-28 weeks instead of 14-22weeks.

Conclusion: Detailed findings of this study could aid the embryologists, neonatologists and nephrologists to understand the chronology of nephrogenic events and related consequences of developmental abnormalities.

Key words: Nephrogenic Events, Human embryos, Foetal Kidney, Pronephric, Mesonephric, Metanephric, Phylogeny.

Address for Correspondence: K. Thyagaraju, Assistant Professor, Department of Anatomy, Sri

Padmavathi Medical College for Women, SVIMS University, Tirupati, Andhra Pradesh, India.

E-Mail: tyagaraj21@gmail.com

Access this Article online

\section{Quick Response code}

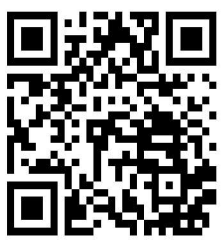

DOI: $10.16965 /$ ijar.2018.369 Journal Information

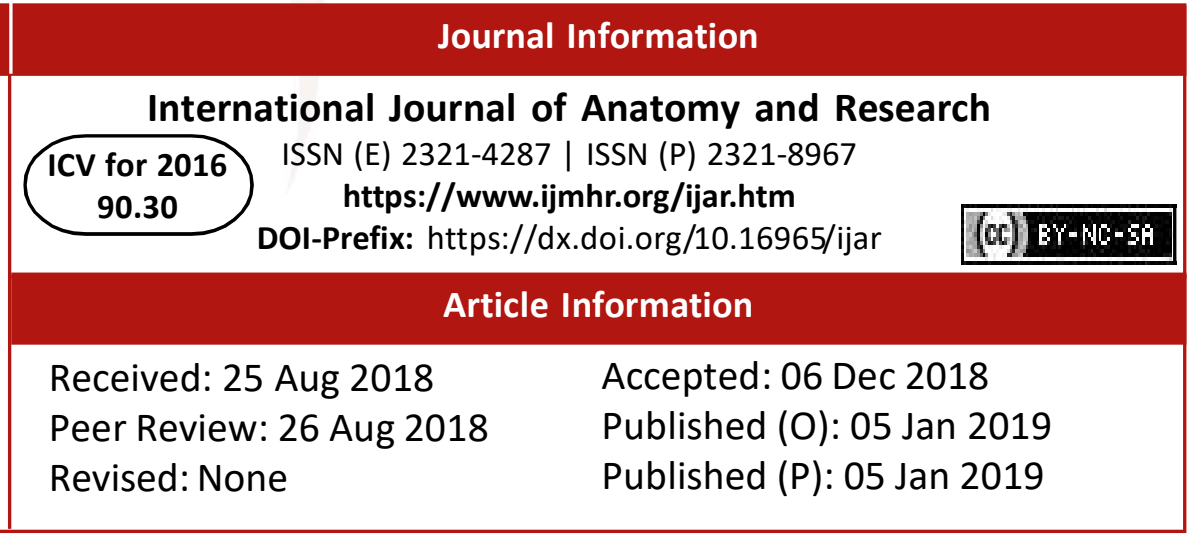




\section{INTRODUCTION}

Kidney is one of the five vital organs of the body for survival, the other being the brain, heart, lung and liver. The basic functions of kidney are regulation of $\mathrm{pH}$ and ion concentration of blood, osmolarity and volume of extracellular fluid, elimination of waste products and toxins and production of hormones.

The crucial stage in human development is the pre-natal period. The embryo or foetus is a collection of genetic scriptures written at different times during intrauterine life under different environmental, nutritional and hereditary conditions. Maternal malnutrition influences the growth and development of foetus leading to foetal undernourishment that dictates foetal vascular, endocrine and metabolic readjustments. This results in low birth weight and can bring out permanent structural and functional changes that can manifest before birth or at some point in the postnatal life. Genetic predisposition to certain diseases with added adverse life style can lead to low birth weight and increased chances for adult onset diseases like hypertension, diabetes etc. Life long expression of genetic patterns for various diseases are set into motion during the critical periods of development and in some instance, they are passed to the future generations. It can be said that "how we are guided into life determines how we are going to live and leave our life".

Normal development of the human kidney is a highly complex process that can go wrong more commonly. Congenital malformations of kidney include its immaturity, absence and poor development that are responsible for long term kidney failure at a very young age requiring dialysis and transplantation. Genetic mutations especially in transcription factors or prenatal exposure to teratogens and maternal malnutrition can impair prenatal development of kidney. Understanding the various mechanisms in the pathogenesis of renal diseases is important for prenatal diagnosis of renal anomalies, protection from teratogens, genetic counselling and for planning medical and surgical therapeutic methods.

The development of kidney is cited as an example for "ontogeny repeats phylogeny" as during its development from intermediate mesoderm the human kidney passes through the three stages as observed in lower mammals. These three stages are pronephros, mesonephros, and metanephros. As the structural and functional units of kidney, the nephrons are the one that pass through these evolutionary stages it is also called nephrogenesis (Fig. 1) [1].

The pronephros and mesonephros degenerate during fetal life while the metanephros develops into the adult human kidney. The anatomy of these structures was described in the literature by several authors [2-6]. A time table of important events during kidney development and morphological events are described by Vize et.al (2003) $[7,8]$.

The human pronephros appears at the 10-somite stage on day 22 of gestation (i.e., 22 days after fertilization), which is morphologically equivalent to embryonic day 9 in mice. Initially, it consists of a small group of nephrotomes with segmental condensations, grooves, and vesicles between the second and sixth somite's. Nephrotomes are nonfunctional, probably representing vestiges of the pronephric kidney of lower vertebrates. This pronephric stage is essential for subsequent kidney development. The pronephric duct that develops from the intermediate mesoderm lateral to the notochord, elongates caudally to reach the cloacal wall on day 26, and is then renamed as the mesonephric, or Wolffian duct, as mesonephric tubules develop. The nephrotomes and pronephric part of the duct involute and cannot be identiûed by 24 or 25 days gestation.

\section{MATERIALS AND METHODS}

Thirty- five aborted embryos and dead foetuses of 5 weeks gestational age to full term were utilized for this study. The entire specimens were preserved in formalin after recording the weight, $\mathrm{CR}$ length and $\mathrm{CH}$ length. Kidneys were removed from the foetuses of more than 8 weeks gestational age by opening the abdominal cavity. The specimens were subjected to routine tissue processing and H\&E staining. 5 embryos of less than 8 weeks gestational age were processed as a whole and were serially sectioned. The histological sections were observed for the time of appearance of various nephrogenic components 
and photographed.

\section{OBSERVATIONS}

Less than 12 weeks GA group: In this group a total of four embryos (less than 8 wks GA) and two fetuses were observed for renal histogenesis.

5 wks. embryo: The youngest embryo observed in this group was that of 5 wks. GA with a CRL of $0.9 \mathrm{cms}$. In this embryo urogenital mesentery, degenerating pronephros and pronephric tubule could be identified (Fig.2). Pronephric tubule was opening in to the coelomic cavity. There was no glomerulus at this stage (Fig.2). A detailed observation at higher magnification (10x) presented renal, gonadal and supra renal primordia along with pronephric and parameson-ephric ducts (Fig.3). In the same slide degenerating pronephros, mesonephros, mesonephric tubules, pronephric duct and cloaca were identified (Fig.4).

6 wks. Embryo: In the 6 wks. GA ( $1.2 \mathrm{cms} C R L)$ embryo urogenital mesentery containing developing mesonephric kidney and gonad were identified (Fig.5). Condensation of metanephric mesenchyme along with mesentery could be identified at this stage.

Growth and differentiation of mesonephros with degenerating mesonephric tubules, mesonephric and paramesonephric ducts and renal corpuscles were seen (Fig.6). Degenerating mesonephric tubules, cut sections of mesonephric duct (MND), paramesonephric duct (PMND), differentiating renal corpuscle (RC) presenting vesicle and ' $S$ ' stages of mesonephric nephron showing continuity with proximal convoluted tubule (PCT) were observed at $6 \mathrm{wks}$. GA.

Change in the pattern of metanephrogenesis from linear to radial fashion could be recognized in the metanephric kidney (Fig.7). Further, interaction between the two functional components i.e. ureteric bud (ductogenesis) and metanephric mesenchyme known as mesenchymal epithelial transition (MET) and morphogenesis could be observed (Fig.8). The various stages in nephrogenesis i.e. interaction, cap, blastemal, vesicle, comma and renal corpuscle stages could be identified (Fig.9)
8 wks. embryo: At 8 wks. GA ( $1.5 \mathrm{cms} C R L)$ differentiating renal corpuscles, proximal and distal convoluted tubules and collecting ducts could be identified (Fig.10). Up to 8 weeks gestational age the sex of the embryo could not be identified.

12 weeks foetus: At 12 week $(2.0 \mathrm{cms} C R L$, male foetus) mesonephric components could not be identified. There is central to peripheral progression in metanephric kidney development and there is no cortico-medullary differentiation of functional components (Fig.11).

13-24 wks GA group: In this group a total of nine foetuses were observed for renal histogenesis. Sections from a specimen of 16 weeks and that of 24 weeks were compared for the developmental progression. During this period cortico-medullary differentiation was observed. At 16 weeks there is increase in the size of the kidney and lobulation was visible. Differentiation of cortex and medulla could be identified. Growing renal pyramid, renal papilla and renal columns could be identified at 16 wks. and 24 wks. (Fig.12). Differentiation of developmental components proceeded from deep cortex to the periphery. Cortex presented three zones. From periphery to centre they are nephrogenic zone presenting immature renal corpuscles, intermediate zone of differentiating renal corpuscles and juxtamedullary differentiated renal corpuscles (Fig.13). Medullary rays and collecting ducts are well formed and are dividing.

Capsule was well differentiated with numerous collagen fibres running parallel to the long axis of the kidney. Beneath the capsule many developing glomeruli were seen in the nephrogenic zone which were now compactly arranged due to decrease in the interstitial tissue (Fig.13). Another important finding in this group was that the parenchyma of the two adjacent lobules was separated by the nephrogenic zone. This zone is similar to the nephrogenic zone seen under the capsule and extends from the subcapsular area to the pelvis of the kidney. It gives the appearance of a column or septum and is known as the primary columns Bertini (Fig $12 \mathrm{~A}$ ). This column marks the junction of primary lobules. Pelvis became extended and many minor calyces open into it. The epithelium lining the 
pelvis at this stage was multi-layered.

Branching and differentiating collecting ducts and undifferentiated tubules at 16 weeks and differentiated tubules at 24 weeks could be identified (Fig.14). Stratified collecting ducts at 16 wks. and differentiating Henle's loops at 24 weeks were observed (Fig.15).

More than 25 weeks GA group: Nephron Induction and differentiation were observed at 28 weeks (Fig.16). Well differentiated Renal corpuscles, glomerulus, tubules, collecting ducts and Henle's loops could be identified at 28 wks. GA (Fig.17 and 18). Comparison of sections of kidney at 28 and 32 weeks presented juxta-cortical ampullary arcades at 28 wks (Fig.19) and degenerating renal corpuscles at 32 wks. At 40 weeks there is disappearance of nephrogenic zone with continued renal vesicle differentiation and interstitial growth (Fig.20).

Fig. 1: Stages of Nephrogenesis.

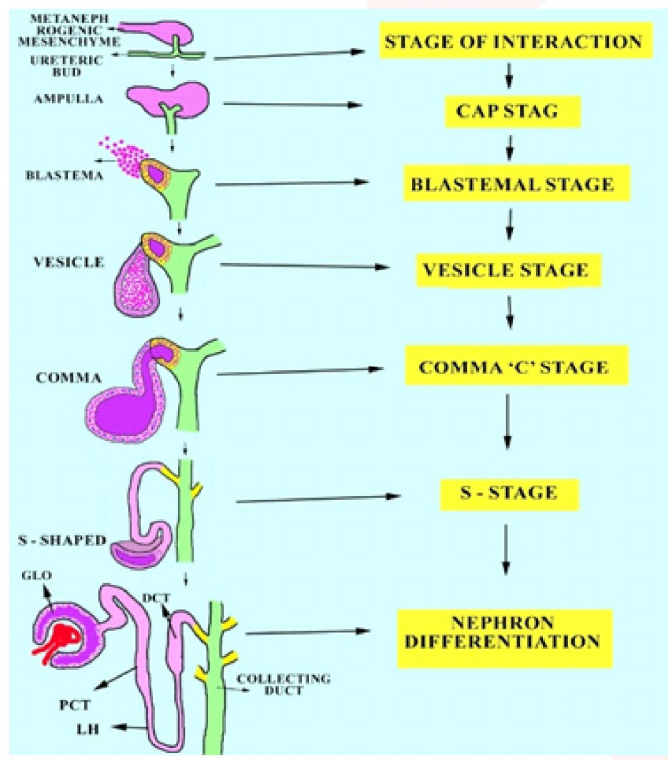

Fig. 2: 5 weeks (0.9 CMS CRL) embryo: A. 4x magnification B. $10 \times$ magnification. ( H\&E 10X, 40X)

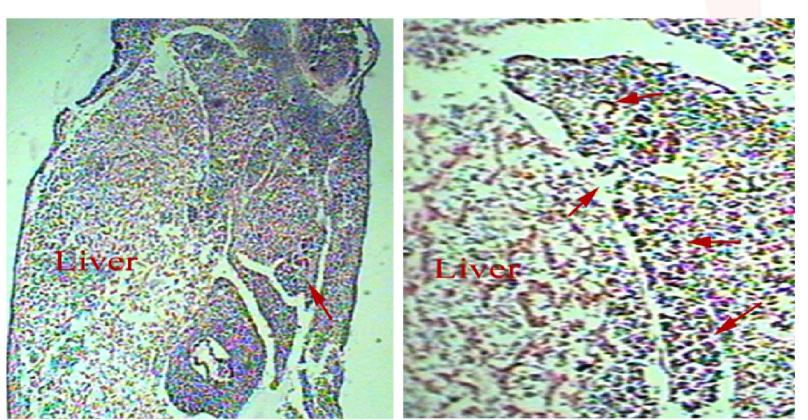

Degenerating pronephros, Urogenital mesentery, Degenerating Pronephric tubule opening in to coelomic cavity (arrows) pronephric tubule, No glomerulus
Fig. 3: 5 weeks - 0.9 CMS CRL -13- 15 stage. (H\&E 10X, $40 \mathrm{X})$

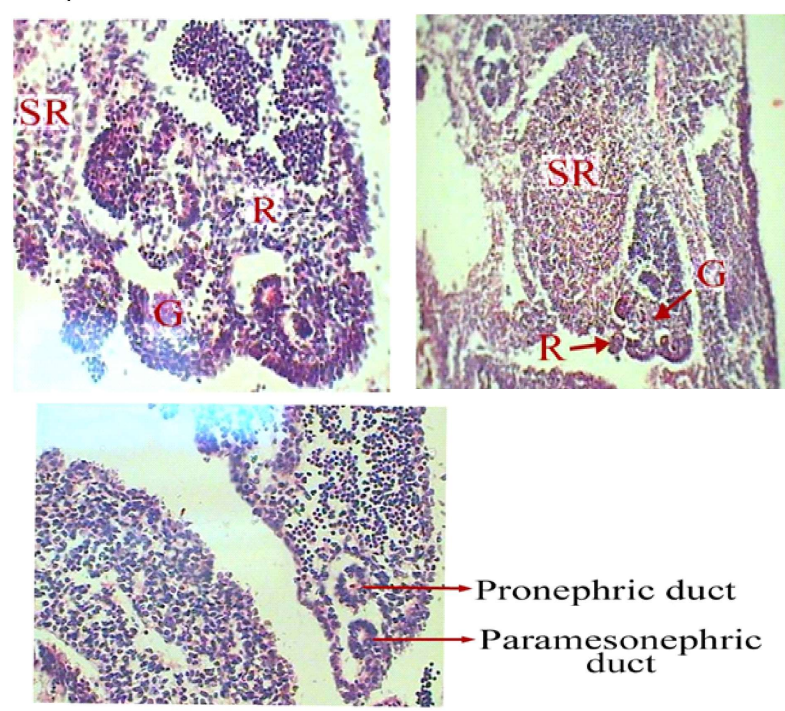

Renal primordium (R), Gonadal primordium (G), Supra renal primordium (SR)

Fig. 4: 5 WEEKS - 0.9 CMS. ( H\&E 10X, 40X)
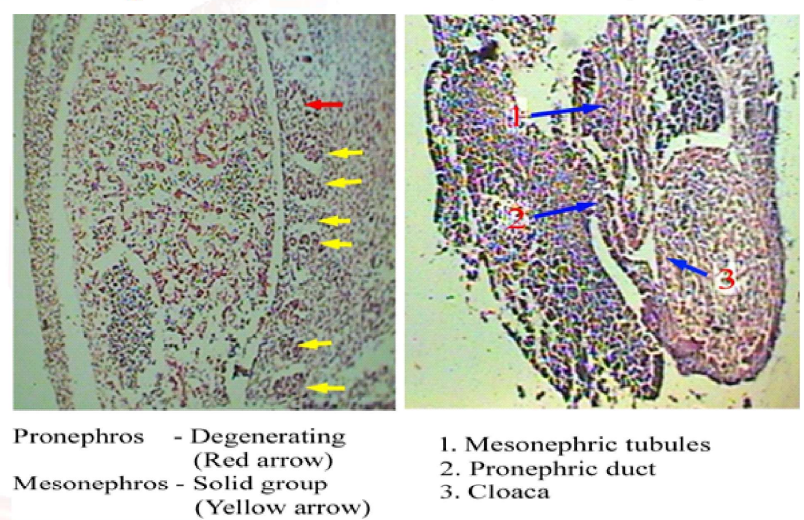

Fig. 5: 6 WEEKS - 1.2 CMS CRL -16/17 STAGE. ( H\&E 10X)

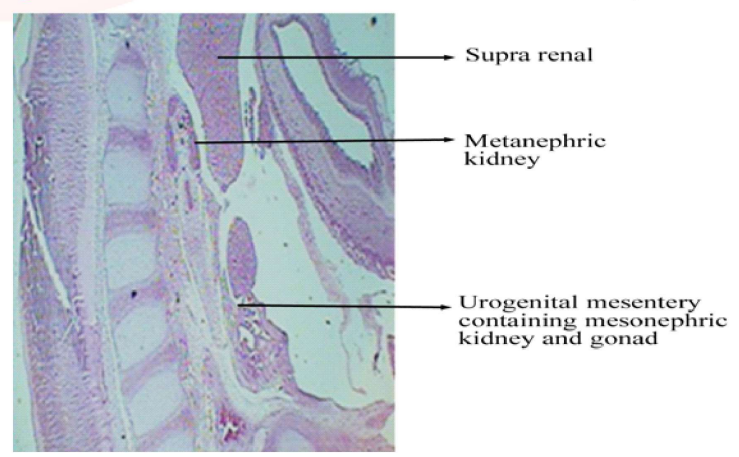

Fig. 6: 6 WEEKS - 1.2 CMS CRL -16/17 STAGE (Growth and differentiation of mesonephros ( H\&E 10X, 40X)

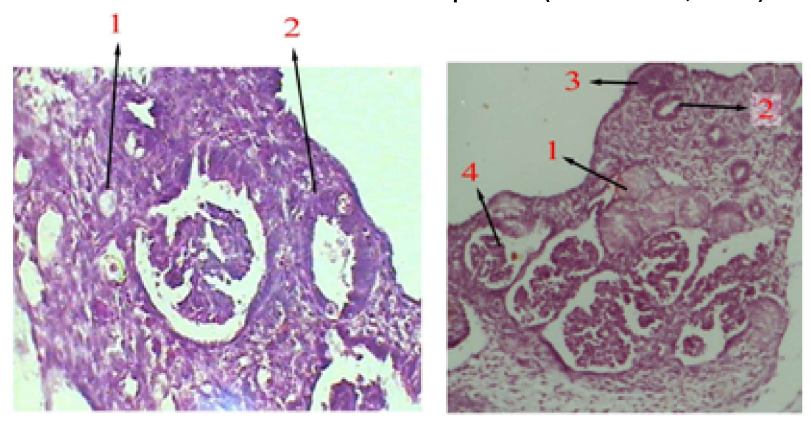

1. Degenerating Mesonephric tubules, 2. MND, 3. PMND, 4. Renal corpuscles 
Fig. 7: 6 WEEKS - 1.2 CMS CRL -16/17 STAGE (Metane phrogenesis - Development changes from linear to radial fashion.) ( $H \& E$ 10X, 40X)

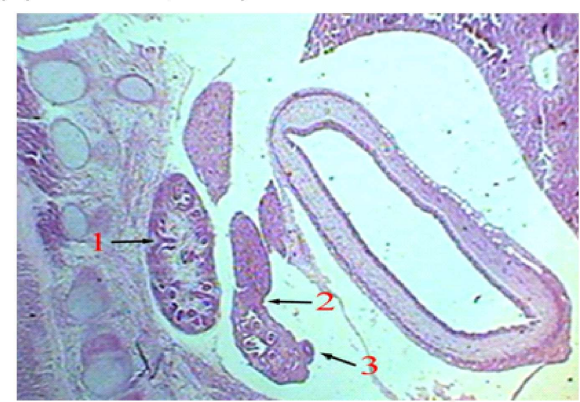

1. Metanephric kidney, 2. Urogenital mesentery 3. Mesonephric kidney 4. Mesonephric duct Fig. 8: 6 WEEKS - 1.2 CMS CRL -16/17STAGE Interaction between two functional components ( H\&E 10X, 40X).

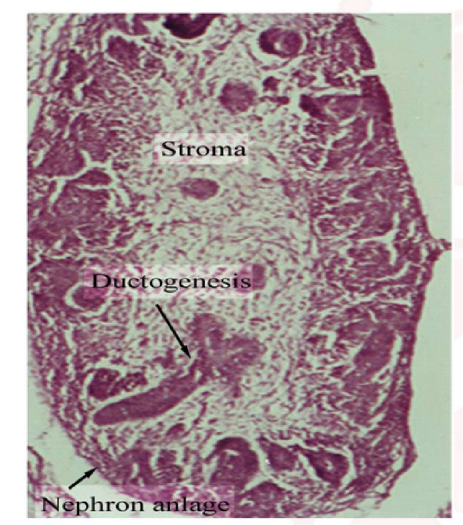

Fig. 9: 6 WEEKS - Metanephric kidney MET and morphogenesis ( H\&E 10X, 40X).

1. Interaction Stage UB and MNM,

2. Cap formation Ampulla + MNM,

3. Blastemal stage 4. Vesicle stage, Comma stage, Renal corpuscle

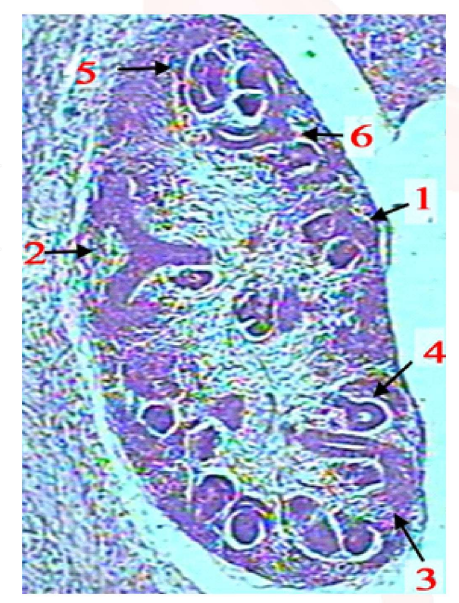

Fig. 10: 8 weeks - $1.5 \mathrm{cms}$ CRL - 18- 23 stage (Differentiating Renal Corpuscle, tubules, duct) ( H\&E 10X, 40X).

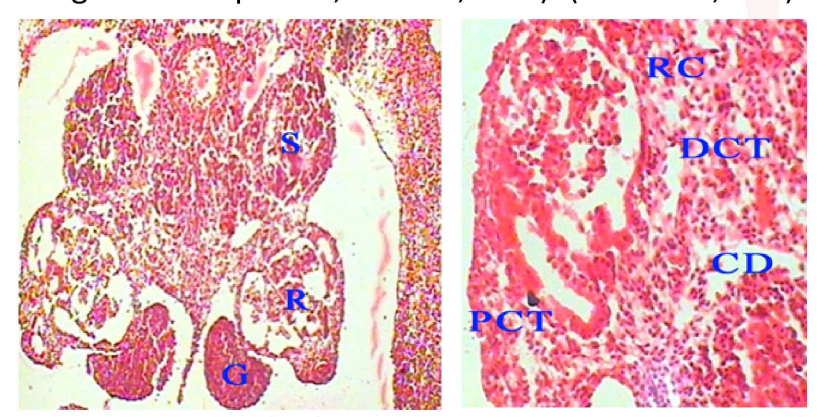

Renal corpuscle (RC) Proximal convoluted tubule (PCT) Distal convoluted tubule (DCT) Collecting Duct (CD) INDIFFERENT GONAD STAGE
Fig. 11: 12 weeks - Male - $2.0 \mathrm{cms}$ CRL No mesonephric component, central to peripheral progression in metanephric kidney, No CM differentiation of functional components. ( H\&E 10X, 40X)

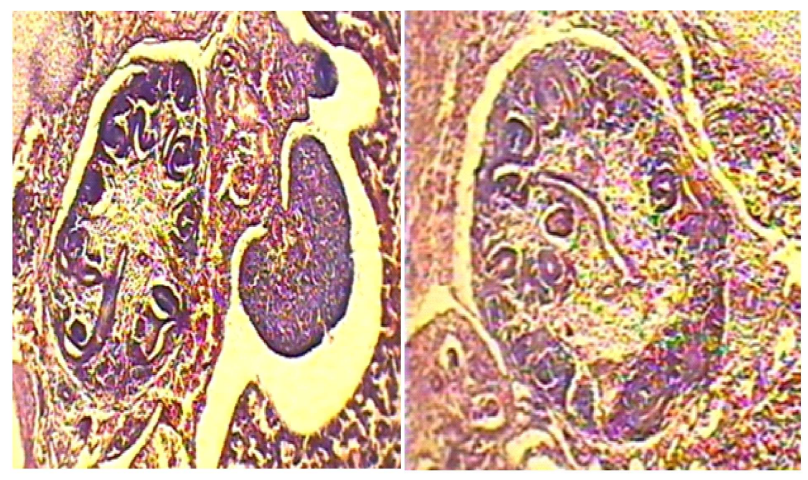

Fig. 12: 16 weeks and 24 weeks GA kidney Cortico - Medullary differentiation.
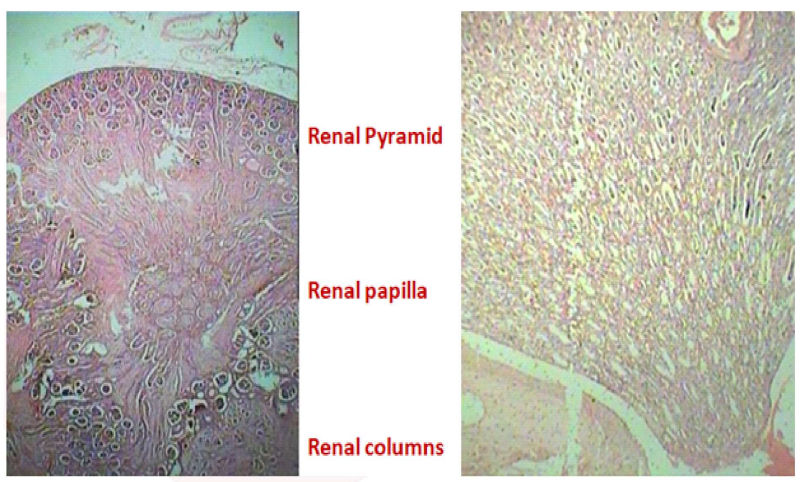

Fig. 13: 16 weeks and 24 weeks GA Differentiation of developmental components proceeded from deep cortex to periphery. ( $H \& E$ 10X)

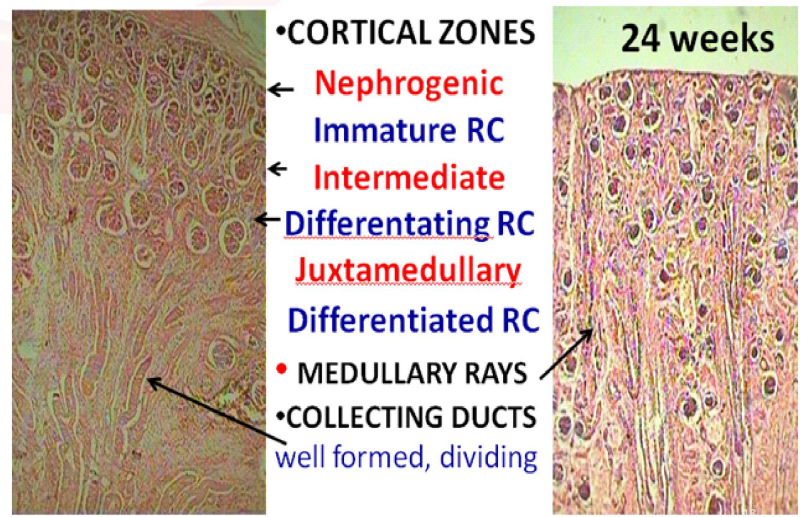

Fig. 14: 16 wks - 24 weeks. ( H\&E 10X, 40X). Branching and differentiating Collecting ducts Undifferentiated tubules
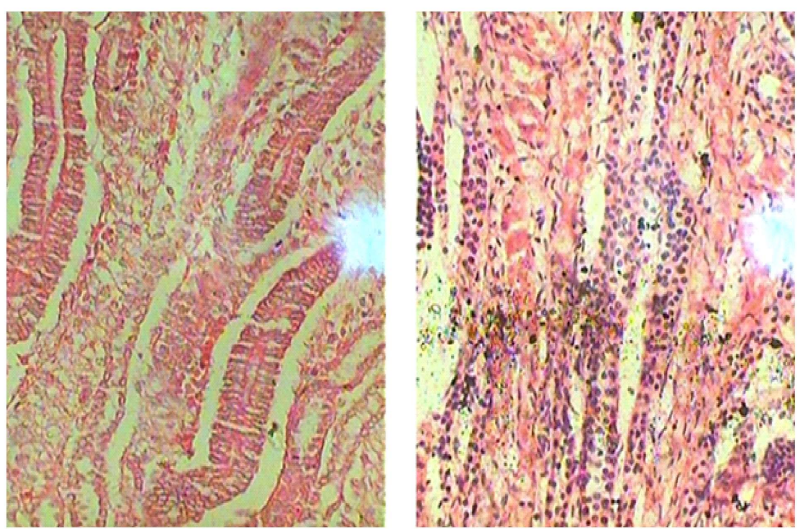

6030 
Fig. 15: 16 weeks - 24 weeks ( H\&E 10X, 40X).

Stratified CD's, Henle's loops differentiating
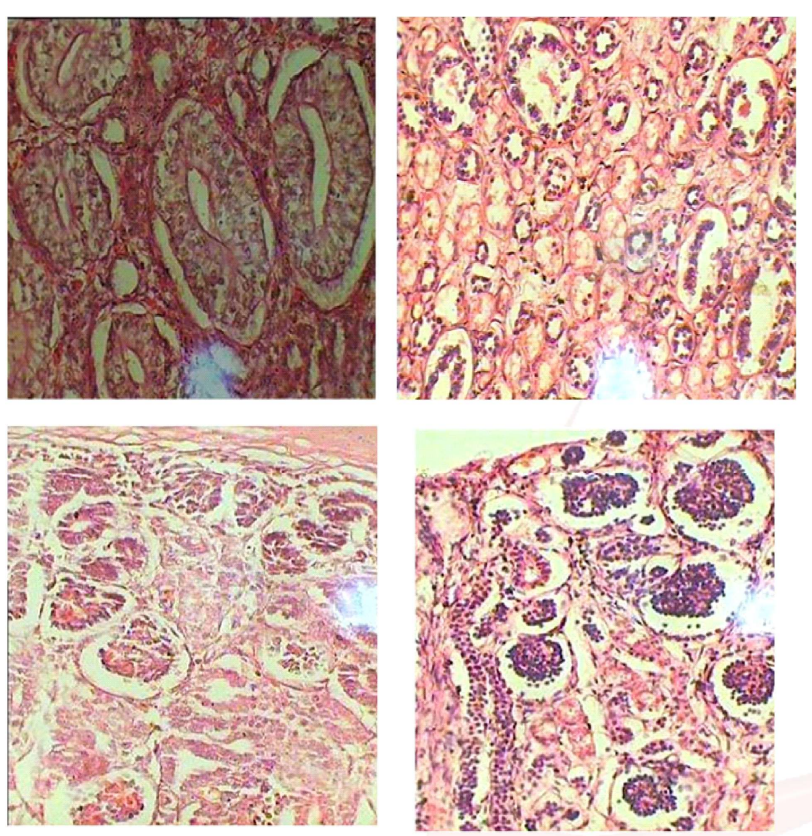

Fig. 16: 28 weeks Nephron induction and differentiation continued. ( H\&E 10X, 40X)
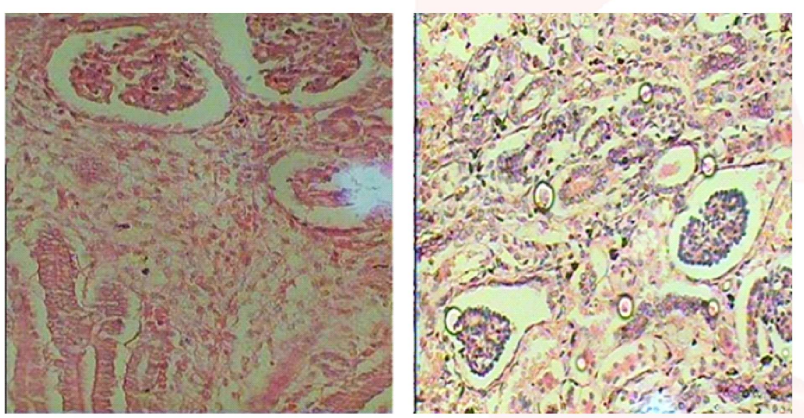

Fig. 17: 28 weeks Well differentiated Renal corpuscles, glomerulus ,tubules, and Collecting ducts. ( H\&E 10X, 40X)
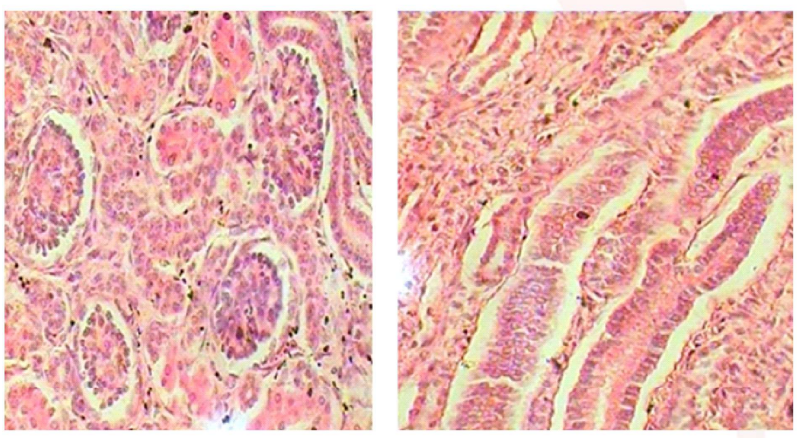

Fig. 18: 28 weeks Well differentiated collecting ducts and Henle's loops. ( H\&E 10X, 40X)

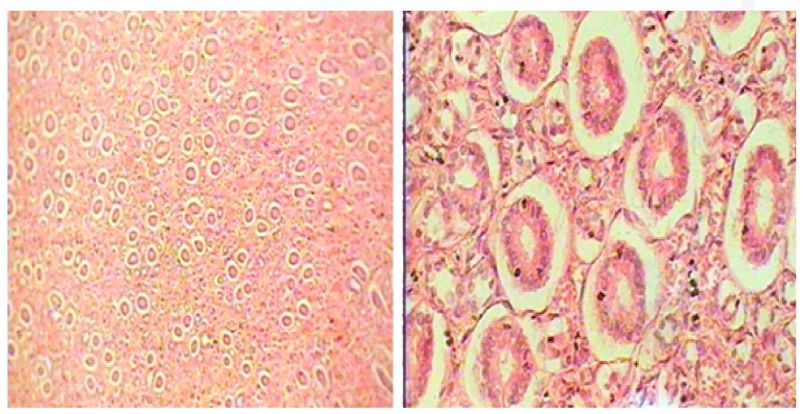

Fig. 19: 28 weeks - 32 weeks Juxta-cortical. ( H\&E 10X, 40X)

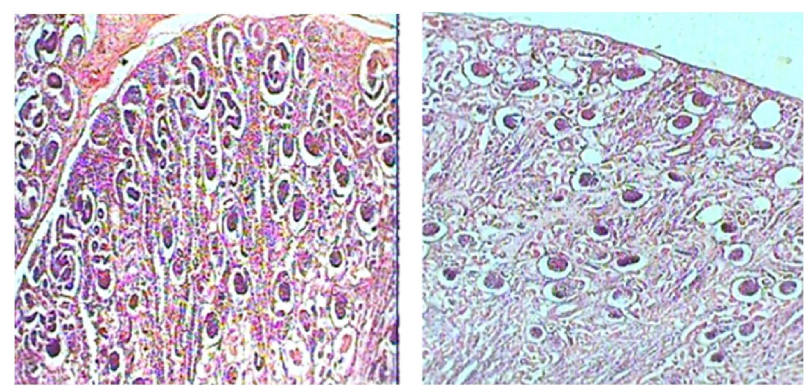

Ampullary arcades, Nephrogenic zone +, Degenerating RC

Fig. 20: 40 weeks Disappearance of nephrogenic zone, Vesicle differentiation continued, Interstitial growth Renal corpuscle, PCT, DCT, Loops of Henle. ( H\&E 10X, 40X)
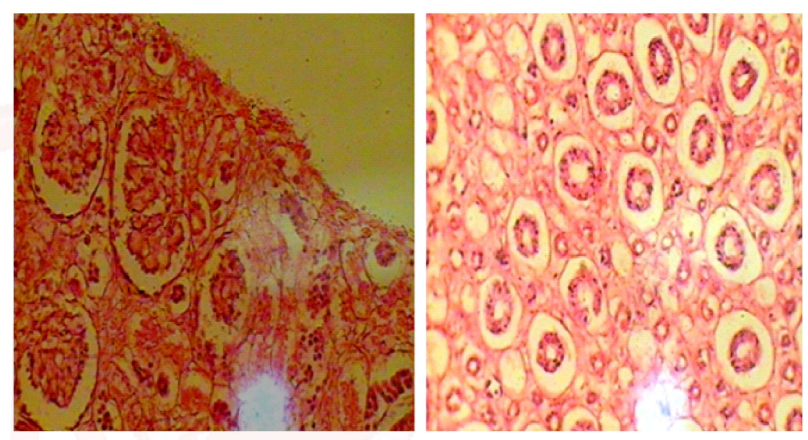

\section{DISCUSSION}

According to Hamilton, Mossman and Boyd textbook of embryology the renal morphogenesis starts at $5^{\text {th }}$ week of gestation and extends up to last month of last trimester of pregnancy. But, there were no detailed histological depictions of microscopic observations on developing human kidneys. All interpretations were based on animal studies. As per the literature all three stages of nephrons appear during $5^{\text {th }}$ week. But, in our study pro and mesonephric stages were visualized simultaneously at $5^{\text {th }}$ week and meso and metanephric stages at $6^{\text {th }}$ week [9].

In the literature the earliest description on nephrogenic events in human was that at 10 weeks GA [10]. In the present study the nephrogenic events in the $5^{\text {th }}, 6^{\text {th }}$ and $8^{\text {th }}$ weeks of embryonic period were observed that facilitated observations on certain of the events that were reported in animal studies. The degenerating pronephros, Urogenital mesentery, pronephric tubule, pronephric tubule opening in to coelomic cavity, absence of glomerulus, presence of Renal $(R)$, gonadal (G), and Supra renal (SR) primordia, pronephric and paramesonephric ducts, 
mesonephric tubules and cloaca were observed at 5 weeks $G A$ and could be described in detail that was not reported in literature for human embryos [10].

In the present study at 6wks GA metanephric kidney, urogenital mesentery containing mesonephric kidney and gonad, degenerating mesonephric tubules, mesonephric and paramesonephric ducts, renal corpuscles, ' $\mathrm{S}$ ' and vesicle stages of metanephrogenesis were observed. Mesenchymal epithelial transition (MET) and various stages in nephrogenesis i.e. interaction, cap, blastemal, vesicle, comma and renal corpuscle could be identified as early as $6^{\text {th }}$ week. A similar description of interaction between ampulla of ureteric bud and adjacent metanephric tissue was reported at 10 weeks by Shalika sharma and Sunanda Raina [10].

Bhattam Narasinga Rao and Mantraratnam Pramila Padmini (2012) [11], reported Invagination of nephric vesicle to form S or V-shaped bodies and glomerulus at $14^{\text {th }}$ week of gestation, whereas the same were observed at 6 weeks in the present study. Takano et.al., (2007) [12] observed V shaped at 13-19 wks and ' $\mathrm{S}$ ' shaped glomeruli at $20-24$ weeks. According to Maria et al (2002) [13] nephrogenesis in human starts at 6th week of intrauterine life and is completed by 35th week of gestation which is similar to our findings. Differentiation of nephron was not complete at 6 weeks though quoted in the literature.

Cortico-medullary differentiation, primary columns of Bertini, was observed at 16 wks GA. Sabita et.al. [14] and Bhattam narasinga rao reported cortico-medullary transition at $16 \mathrm{wks}$ which was similar to the observation in the present study. Shalika sharma and Sunanda Raina [10] reported the same at 14 weeks. According to Hamilton, Mossman and Boyd this appears at 12 weeks GA [9]. Hosapatna $M$ et al (2015) [15] also did not observe distinct differentiation of cortex and medulla until 12th week. Anant Dinesh et.al., (2006) reported corti co-medullary demarcation at 18-25 weeks [16]. The difference in the time of appearance reported by different studies could be due to racial differences. Nephron arcades were observed at 16 weeks in the present study while the same was reported at 16-18 weeks by Shalika sharma and Sunanda raina [10]. Medullary rays could be observed at 24 weeks in the present study and were reported at 28 weeks by Shalika sharma and Sunanda raina. According to Sadiqali, et al. (2012) [17] and Hosapatna M, et al. (2015) [15] at 14 weeks of gestation the section of kidney showed a nephrogenic zone containing undifferentiated mesenchymal cells just underneath the capsule and their thickness decreased at 32 weeks. In the present study the nephrogenic zone was observed at 16 weeks and was absent at 40 weeks.

In 8 wks embryo differentiating Renal corpuscle $(\mathrm{RC})$, Proximal convoluted tubule (PCT), Distal convoluted tubule (DCT), Collecting Duct (CD) could be identified in the present study. At 12 week there is central to peripheral progression in metanephric kidney development and there is no cortico-medullary differentiation of functional components. According to Tank, et al. (2012) [18] at 12 weeks the kidney was covered by a thin capsule with an undifferentiated corticomedullary junction. Shalika Sharma, Sunanda Raina (2014) [10] reported very small and undifferentiated renal pelvis at 10 weeks and fully differentiated at 18 weeks. They reported Zone of transition between cortex and medulla starting at 14 weeks, presence of lobulation as early as 10 weeks and lobular fusion at 15 weeks of gestation. In our study these were observed at 16 weeks GA.

According to Maria et al (2002) [13], Mishra S, et al. (2006) [19], Sadiqali, et al. (2012) [17] and Hosapatna M, et al. (2015) [15] corticomedullary differentiation occurs between 20- 24 weeks. According to Maria $\mathrm{H}$ et al (2002) the corticomedullary differentiation completes between 25-30 weeks [13]. Similar findings were observed in the present study.

By 40 wks GA disappearance of nephrogenic zone and continuation of vesicle differentiation was observed which was similar to that reported by Sadiqali, et al. (2012) [17] and Hosapatna M, et al. (2015) [15]. According to DakoviæBjelakoviæ, et al. (2005) [20] in the superficial part of the cortex, nephrogenic zone was very large at lower weeks of fertilization but as weeks of fertilization increased size of nephrogenic zone decreased and it was absent at 36 weeks of fertilization. Shimada K et al (1993) [21]. 
Observed nephrogenic zone in all kidneys before 34 weeks of gestation, and disappeared in all kidneys after 37 weeks. Potter L (1965) [2]. Tank K. C. (2012) [18], Morag S (1959)[22] in their study observed the disappearence of nephrogenic zone by 36th week where as In the present study the it was observed till 32 weeks.

\section{CONCLUSION}

When compared to literature we observed two weeks delay in the appearance of pro and mesonephric components that were observed at $5^{\text {th }}$ week instead of $3^{\text {rd }}$ and $4^{\text {th }}$ weeks. Meso and metanephros appeared during the 6 th week instead of 4th and 5th weeks. Differentiation of other components has not completed by 6th week.

There is delay in further differentiation of metanephric components. Cortico-medullary differentiation (16 wks. Vs 12 wks.), demarcation of renal pelvis (16 wks. Vs 10-12 weeks), Pelvi-calyceal system (16 weeks Vs 12-14 weeks), morphologically recognizable nephrons (16 weeks Vs 12-14 weeks) and stage of complete differentiation of metanephric components (24-32 weeks).

Major part of development was observed between 16-28 weeks instead of 16-24weeks as reported in literature. Ampulla division continued beyond 24 weeks, increased number of mature nephrons are seen between 24-28 weeks instead of 16-20 wks. Nephron arcades were observed between 24-28 weeks instead of 1422 weeks when compared with literature, delay of 2 to 6 weeks in the chronology of appearance/disappearance/differentiation/maturation of various morphological components in the ontogenesis of metanephric kidney while recapitulating its ancestral history (phylogeny) were observed in the present study.

\section{ACKNOWLEDGEMENTS}

This paper is dedicated to Late Dr. Ch. Vidyavathi MD Anatomy, Ex. Associate Professor, Government Medical College, Guntur, Andhra Pradesh, India. Remembering her efforts in collecting some of the samples and processing for histology.

\section{Conflicts of Interests: None}

\section{REFERENCES}

[1]. Subhadra devi V. Inderbir Singh's Human embryology, Jaypee Brothers Medical Publishers; 11th edition (2018).

[2]. Potter L. Development of the human glomerulus. Archives of pathology, 1965;80: 241-255.

[3]. Gary C. Schoenwolf, Philippa H. Francis-West, Steven B. Bleyl, Philip R. Brauer Editors: Philip R. Brauer, Steven B. Bleyl, Gary C. Schoenwolf. Larsen's Human Embryology, Churchill Livingstone; 5 edition (December 24, 2014)

[4]. Scott F. Gilbert. Developmental Biology. Sinauer Associates Inc (1887).

[5]. Moore and Persaud, The Developing Human, 10th Edition Saunders Title 2016.

[6]. Woolf AS, Winyard PJ, Hermanns MH, Welham SJ. Maldevelopment of the human kidney and lower urinary tract: an overview. In The kidney 2003:377393.

[7]. Peter D. Vize, Adrian S. Woolf, Jonathan B.L. Bard. The Kidney: From Normal Development to Congenital Disease. Elsevier, 2003.

[8]. Vize PD, Carroll TJ, Wallingford JB. Induction, development, and physiology of the pronephric tubules. InThe Kidney 2003;19-50.

[9]. Harland W. Mossman and James W. Hamilton. Human Embryology: Prenatal Development of Form and Function 1945.

[10]. Shalika Sharma, Sunanda Raina. STUDY OF HUMAN FETAL KIDNEY. Int J Anat Res 2014;2(4):785-790. DOI: 10.16965/ijar.2014.550.

[11]. Bhattam Narasinga Rao and Mantraratnam Pramila Padmini. PRENATAL HISTOGENESIS OF KINDEY IN HUMAN FOETUSES. International Journal of Basic and Applied Medical Sciences. 2012; 2(2):144-147.

[12]. Takano K, Kawasaki Y, Imaizumi T, Matsuura $H$, Nozawa R, Tannji M, Suyama K, Isome M, Suzuki H, Hosoya M. Devlopment of glomerular endothelial cells, podocyte and mesangial cells in the human fetus and infant; Tohoku Journal of Experimental Medicine 2007;212(1) 81-90

[13]. Maria H., Daudt L. Diagnosis of the human fetal age based on the development of the normal kidney. Journal Brasileiro de Patologia e Medici Laboratorial., 2002; 38(2): 1-10.

[14]. Sabita mishra, Anant Dinesh, J.M. Kaul, Morphological and morphometrical study of human renal development during midgestational period, JASI. 2006; 55(2): 5-10.

[15]. Hosapatna M, Bangera H, D Souza A, Das A, Supriya, D Souza AS, Ankolekar VH. Histological differentiation of human fetal kidney. IAIM, 2015; 2(7): 49-54.

[16]. Anant Dinesh, Anant Mishra and Kaul J.M. Morphological and Morpho-metrical Study of Human Renal Development During Mid-Gestation Period. Journal of Anatomical Society of India 2006;55(2):07 12. 
[17]. Syed Sadiqali. A., Joshi R. A., Herekar N. G. Histogenesis of Kidney in Human Fetuses. International Journal of Recent Trends in Science And Technology, 2012; 3(2): 44-48.

[18]. Tank K. C., Saiyad S. S., Pandya A. M., Akbari V. J., Dangar K. P. A study of histogenesis of human fetal kidney. Int J Biol Med Res., 2012; 3(1): 1315-1321.

[19]. Mishra S., Dinesh A., Kaul J. M. Morphological and morpho-metrical study of Human renal development during midgestational period. J. Anat.soc. India, 2006; 55(2): 5.

[20]. Dakoviæ-Bjelakoviæ M., Cukuranoviæ R., Antiæ S., Bjelakoviæ G., Mitiæ D. Quantitative analysis of the nephron during human fetal kidney development. Vojnosanit Pregl., 2005; 62(4): 281-286.
[21]. Shimada K, Hosokawa S, Tohda A. Histological study of the fetal kidneys. Development of the nephrons according to gestational weeks. Nihon Hinyokika Gakkai Zasshi. 1993 Dec;84(12):2091-6.

[22]. Morag S., Emery J. L. The late intrauterine and postnatal development of human renal glomeruli. Journal of Anatomy, 1959; 93(3): 331-340.

[23]. Khayati Sant Ram, Anshu Sharma, Mahesh Sharma, Naresh Goel, Daisy Sahni. HISTOGENESIS OF FETAL KIDNEY IN FETUSES OF DIFFERENT GESTATIONAL AGE GROUPS. J. Anat. Sciences, 2015;23(1):1-4.

How to cite this article: Subhadra Devi Velichety, K. Thyagaraju, Siva Kumar Vishnubhotla, B. Ravindra Kumar. CHRONOLOGY OF NEPHROGENIC EVENTS IN STAGED ABORTED HUMAN EMBRYOS AND FETUSES. Int J Anat Res 2019;7(1.1):6026-6034. DOI: 10.16965/ijar.2018.369 